\title{
Percepção dos usuários e profissionais de saúde no Distrito Federal: os atributos da atenção primária
}

\author{
Perception of users and health professionals in the Federal District: \\ the attributes of primary care
}

Rosânia de Lourdes Araújo', Ana Valéria Machado Mendonça², Maria Fátima de Sousaㅉ

RESUMO O presente artigo analisa os atributos da Atenção Primária à Saúde em duas regiões do Distrito Federal, focalizando o cuidado integral à criança. Compara-as a partir dos usuários e profissionais de Unidades Básicas Tradicionais e da Estratégia Saúde da Família. O método utilizado foi o qualiquantitativo. Os dados foram coletados por meio de inquérito, através do PCATool-Brasil, aplicado a profissionais e usuários. Os participantes apontaram insatisfação com o cuidado à saúde integral da criança nas duas regiões. O acesso, a longitudinalidade, a integralidade e a coordenação são limitados na teoria e na prática dos profissionais, nos dois modelos de atenção. Ambos demandam aprofundamento dos processos organizativos da saúde integral da criança.

PALAVRAS-CHAVE Atenção Primária à Saúde; Estratégia Saúde da Família; Integralidade em saúde.

\begin{abstract}
The present article analyses the attributes of Primary Health Care in two regions of the Federal District, focusing on integral care to child. It compares them from users and professionals of Traditional Basic Units and Family Health Strategy. The method used was quali-quantitative. Data were collected by means of survey, through the PCATool-Brazil, proposed to professionals and users. Participants pointed out dissatisfaction with the care to the integral health of the child in the two regions. Access, longitudinality, entirety and coordination are limited in theory and practice of the professionals, in the two models of attention. Both demand deepening the organizational processes of the integral health of the child.
\end{abstract}

1 Universidade de Brasília (UnB) - Brasília (DF),

Brasil.

rosaniaa@gmail.com

2 Universidade de Brasília (UnB), Departamento de Saúde Coletiva - Brasília (DF), Brasil.

valeriamendonca@gmail.

com

3 Universidade de Brasília (UnB), Departamento de Saúde Coletiva - Brasília (DF), Brasil.

fatimasousa@unb.br
KEYWORDS Primary Health Care; Family Health Strategy; Integrality in health. 


\section{Introdução}

No Brasil, vários foram os ciclos sucedidos na tentativa de organizar a Atenção Básica (AB) como base estruturante do modelo de atenção à saúde (MENDES, 2012). Desde a criação do Sistema Único de Saúde (SUS), fortalece-se a ideia de que a Atenção Primária à Saúde (APS) é base estruturante para os sistemas em todos os níveis organizacionais; e, nesta direção, a Política Nacional de Atenção Básica (PNAB), criada em 2006 e atualizada em 2011, estabelece diretrizes e normas a serem seguidas em todo o País (BRASIL, 2012).

De acordo com a PNAB, a APS está definida como um conjunto de ações de saúde, nos âmbitos individual e coletivo, que abrangem a promoção e a proteção da saúde, a prevenção de agravos, o diagnóstico, o tratamento, a reabilitação e a manutenção da saúde. Orienta-se pelos princípios da universalidade, da acessibilidade e da coordenação do cuidado, do vínculo e da continuidade, da integralidade, da responsabilização, da humanização, da equidade e da participação social (BRASIL, 2012).

Para Sousa e Hamann (2009), a APS é uma formulação típica do SUS, que deixa claro os seus princípios e as suas diretrizes organizativas, e é incorporada pelo Programa Saúde da Família (PSF), o qual, a partir de sua criação, materializa uma forma de pensar e agir na construção de um novo modelo de atenção à saúde dos indivíduos, famílias e comunidades. Iniciado em 1994, o PSF passou a ser considerado a principal estratégia da PNAB para a organização e expansão da APS no País (BRASIL, 2012).

A sistematização do conceito de atenção primária, elaborado por Starfield (2002), é considerada um marco referencial para análise da APS em todo o mundo. A autora parte da compreensão da APS como:

[...] aquele nível de um sistema de serviços de saúde que oferece a entrada no sistema para todas as novas necessidades e problemas, fornece atenção sobre a pessoa (não direcionada para a enfermidade) no decorrer do tempo, fornece atenção para todas as condições, exceto as muito incomuns ou raras, e coordena ou integra a atenção fornecida em algum outro lugar ou por terceiros. (STARFIELD, 2002, P. 28).

Com relação aos serviços da APS, é caracterizada a presença de sete atributos, também chamados dimensões, como eixos estruturantes, que estão associados à qualidade, à efetividade e à eficiência de suas intervenções: o acesso, a longitudinalidade, a integralidade da atenção, a coordenação da atenção - considerados atributos primários; a atenção à saúde centrada na família, a orientação para a comunidade e a competência cultural - como atributos derivados. Dessa forma, quanto maior for a presença e a força desses atributos, mais forte é a sua orientação para a atenção primária (STARFIELD, 2002).

A partir dessa base conceitual, Starfield, Xu e Shi (2001) desenvolveram uma ferramenta que permite a mensuração desses atributos, denominada Primary Care Assessment Tool (PCATool). Esse instrumento foi adaptado e validado para a realidade brasileira, denominando-se PCATool-Brasil (BRASIL, 2010).

Estudos nacionais e internacionais constataram o alcance dessas dimensões na avaliação dos serviços de APS (ROMERO; COLLANTES; RAMIREZ, 2009; MACINKO ET AL., 2006; ELIAS ET AL., 2006; IBANEZ ET AL., 2006). Outros estudos concluíram que os escores do PCATool podem ser utilizados como indicadores de qualidade de atenção, contribuindo como um instrumento para investigação dos serviços de saúde, assim como um instrumento de avaliação comparativo do grau de orientação à atenção primária dos serviços de APS no Brasil (HARZHEIM ET AL., 2006; OLIVEIRA, 2007; CHOMATAS, 2009; LEÃO; CALDEIRA; OLIVEIRA, 2011).

A Estratégia Saúde da Família (ESF), como passou a ser denominado o PSF, busca cumprir os princípios da APS definidos por 
Starfield, Xu e Shi (2002), de forma a ser o primeiro contato da população com as ações e os serviços, com integralidade, ao longo do tempo, coordenando os usuários na rede de serviços.

A implantação dessa estratégia, no Distrito Federal (DF), ocorreu em 1997, e, ao contrário do movimento de expansão observado no resto do País, ao longo da sua história, a ESF, no DF, caracteriza-se pela descontinuidade e pela pouca cobertura populacional. Em abril de 2012, quando da realização do estudo, a ESF apresentava uma cobertura populacional em torno de $15,3 \%$. No DF, coexistem os dois modelos de $\mathrm{AB}$, com Unidades Básicas Tradicionais (UBT) e Unidades Básicas de Saúde da Família (UBSF) - estas, presentes em quase todas as Regiões Administrativas (RA), com predomínio nas regiões com menor oferta de serviços de saúde e indicadores de saúde mais desfavoráveis.

Partindo desse entendimento, o estudo analisou os atributos da APS naquilo que afirma Starfield (2002), considerando, assim, os quatro principais atributos: o acesso, a longitudinalidade, a integralidade e a coordenação. Nesse caso, objetivou-se avaliar a presença e a extensão desses atributos no cuidado integral à saúde da criança, comparando as UBT que atuam com o modelo tradicional com as UBSF do DF, na percepção de usuários e profissionais.

\section{Métodos}

Para comparação dos modelos de APS no DF, foram escolhidas duas RA (R1 e R2), devido à predominância nos seus serviços básicos dos dois modelos de atenção primária. A R1 possui a terceira maior população do DF, com 4.989 crianças menores de dois anos, um elevado grau de urbanização e desenvolvimento social e ampla rede de serviços de saúde. A R2 é uma região em expansão, na qual os serviços públicos e sociais ainda encontram-se em processo de estruturação, com uma população de 1.160 crianças menores de dois anos. Para comparação dos modelos de APS, optou-se pela combinação dos métodos quantitativo e qualitativo. Para os dados quantitativos, foi realizado um inquérito por meio do Instrumento PCATool-Brasil (BRASIL, 2010), de acordo com a metodologia validada por Harzheim et al. (2006), aplicado a profissionais médicos pediatras e de família, enfermeiros e cuidadores de crianças. Com relação às UBS, foi considerado todo o conjunto (oito UBT e as cinco UBSF, com 14 equipes da ESF). Para as crianças, foi calculada uma amostra aleatória simples, com erro amostral de $5 \%$ e nível de confiança de $95 \%$. A partir dessa amostra, foram definidos dois estratos homogêneos, segundo as duas RA. Como critério de inclusão, adotou-se a idade 'menor de dois anos', com, pelo menos, um atendimento na UBS, anteriormente.

Para os profissionais das UBT, foram selecionados o enfermeiro da sala da criança e um dos médicos pediatras. Foram excluídos os profissionais da ESF com menos de seis meses de atuação.

Para o estudo qualitativo, foram realizadas entrevistas semiestruturadas, aplicadas a uma subamostra aleatória de 16 profissionais (representantes das oito UBT e das cinco UBSF, acrescidos de mais três profissionais, para igualdade da amostra). Optouse pela avaliação dos atributos considerados essenciais aos serviços de atenção primária. Porém, por entendermos que a presença dos atributos Orientação Familiar e Comunitária, na base organizativa das práticas da saúde da família, no relato dos profissionais, poderia se tornar um viés para a análise comparativa com o modelo tradicional de atenção primária, estes foram desconsiderados na pesquisa qualitativa. O estudo foi realizado no período de junho a setembro de 2012.

O PCATool-Brasil é estruturado em blocos, cada um abrangendo as dimensões e subdimensões da APS, com respostas do tipo Likert, com escala de valores 
preestabelecidos variando de 1 a 4 para cada um dos itens, de maneira que 1 é igual a 'com certeza não', 2 a 'provavelmente não', 3 a 'provavelmente sim' e 4 a 'sim'. Os cálculos dos escores para cada dimensão - para o Escore Essencial, Derivado e para o Escore Geral da APS - foram feitos segundo orientação do 'Manual do PCATool' (BRASIL, 2010). Foram considerados alto escore valores maiores ou iguais a 6,6, o que representa respostas maiores ou iguais a 3 na escala de Likert (OLIVEIRA, 2007).

Para análise dos dados, foi utilizado o aplicativo SPSS for Windows, versão 17.0. Para a comparação das médias de cada atributo entre os tipos de serviços, usou-se o teste ' $t$ ', de Student, com nível de significância de $p<0,05$.

Para os dados qualitativos, usou-se a análise de conteúdo (MINAYO; SANCHES, 1993). Como auxílio para a organização e a codificação das informações, utilizou-se o software QSR Nvivo9, disponibilizado pelo Núcleo de Estudos de Saúde Pública, da Universidade de Brasília.

A pesquisa foi aprovada pelo Comitê de Ética da Fundação de Ensino e Pesquisa em Ciências da Saúde do Distrito Federal (Fepecs-DF) sob número 184/2012.

\section{Resultados e discussão}

Participaram do estudo 34 profissionais, 15 profissionais de UBT e 19 da ESF. Quatro médicos e quatro enfermeiros da ESF foram excluídos devido a licenças médicas, férias ou atuação menor de seis meses na equipe. Uma UBT não possuía enfermeiro na sala da criança. Desses, 16 profissionais (oito por modelo de UBS) participaram do estudo qualitativo.

A amostra foi composta por 387 crianças, sendo que dez foram excluídas por desistência. Foram avaliadas, portanto, 377, sendo 306 acompanhadas pelas UBT e 71 pela ESF. Do total, $66 \%$ eram menores de um ano de idade, e $54,6 \%$ do sexo feminino. A idade média foi de 9,4 meses, não havendo diferença entre os dois estratos de crianças. Os cuidadores/acompanhantes, na grande maioria dos casos, eram as mães (94,2\%). No estrato da R1, os cuidadores tinham entre 26 e 35 anos (47\%), e $52 \%$ com ensino médio completo. Na R2, $47 \%$ a idade era entre 15 e 25 anos, e $22 \%$ possuíam ensino fundamental incompleto.

Observou-se que o conteúdo das falas dos profissionais, no conjunto das abordagens e no contexto da presença e da extensão de cada atributo, circundava a identificação de fatores que contribuíam para facilitar ou dificultar a presença dos atributos nos seus processos de trabalho. A análise dos resultados será apresentada em outro momento. Trechos de fala serão utilizados para dialogar com os resultados obtidos pelo PCATool.

Na tabela 1, são apresentados os escores avaliados para cada uma das dimensões e subdimensões e o escore geral da APS, na percepção dos cuidadores e dos profissionais, por modelo de serviço. Os valores de escores maiores ou iguais a 6,6, em negrito, foram definidos como altos, e os valores menores que 6,6 foram considerados baixos. As diferenças estatisticamente significativas $(p<0,05)$ também estão representadas com o asterisco. 
Tabela 1. Dimensões da Atenção Primária à Saúde segundo cuidadores e profissionais de Unidades Básicas Tradicionais e Estratégia Saúde da Família. Distrito Federal, 2012

\begin{tabular}{|c|c|c|c|c|c|c|c|c|c|}
\hline \multirow[b]{2}{*}{ DIMENSÃO } & & \multicolumn{4}{|c|}{ Cuidadores } & \multicolumn{4}{|c|}{ Profissional } \\
\hline & & $\mathrm{N}$ & Média & $\begin{array}{l}\text { Desvio } \\
\text { padrão }\end{array}$ & $p$ & N & Média & $\begin{array}{l}\text { Desvio } \\
\text { padrão }\end{array}$ & $p$ \\
\hline \multirow{2}{*}{ Afiliação } & UBT & 306 & 5,47 & 2,89 & \multirow{2}{*}{$0,00^{\star}$} & - & - & - & - \\
\hline & ESF & 71 & 6,80 & 3,22 & & - & - & - & - \\
\hline \multirow{2}{*}{$\begin{array}{l}\text { Acesso de primeiro contato - } \\
\text { Utilização }\end{array}$} & UBT & 299 & 8,06 & 1,99 & \multirow{2}{*}{0,25} & - & - & - & - \\
\hline & ESF & 71 & 8,37 & 2,01 & & - & - & - & - \\
\hline \multirow{2}{*}{ Acessibilidade } & UBT & 302 & 5,45 & 2,22 & \multirow{2}{*}{0,75} & 15 & 3,21 & 0,96 & \multirow{2}{*}{0,12} \\
\hline & ESF & 71 & 5,35 & 2,36 & & 19 & 2,75 & 0,65 & \\
\hline \multirow{2}{*}{ Longitudinalidade } & UBT & 306 & 5,75 & 1,68 & \multirow{2}{*}{$0,00^{*}$} & 15 & 7,53 & 1,24 & \multirow{2}{*}{0,64} \\
\hline & ESF & 71 & 6,59 & 1,90 & & 19 & 7,31 & 1,42 & \\
\hline \multirow{2}{*}{$\begin{array}{l}\text { Coordenação - integração de } \\
\text { cuidados }\end{array}$} & UBT & 62 & 5,56 & 3,52 & \multirow{2}{*}{0,74} & 15 & 7,61 & 1,60 & \multirow{2}{*}{0,13} \\
\hline & ESF & 6 & 6,12 & 3,67 & & 19 & 6,69 & 1,83 & \\
\hline \multirow{2}{*}{$\begin{array}{l}\text { Coordenação - Sistemas de } \\
\text { informações }\end{array}$} & UBT & 302 & 8,16 & 1,29 & \multirow{2}{*}{$0,03^{*}$} & 15 & 9,19 & 0,88 & \multirow{2}{*}{$0,04^{\star}$} \\
\hline & ESF & 69 & 8,50 & 1,12 & & 19 & 8,37 & 1,36 & \\
\hline \multirow[t]{2}{*}{ Integralidade - serviços disponíveis } & UBT & 236 & 6,13 & 1,31 & \multirow{2}{*}{$0,00^{*}$} & 15 & 5,97 &, 97 & \multirow{2}{*}{0,07} \\
\hline & ESF & 57 & 4,15 & 1,43 & & 19 & 5,31 & 1,10 & \\
\hline \multirow{2}{*}{ Integralidade - serviços prestados } & UBT & 302 & 6,02 & 3,15 & \multirow{2}{*}{0,63} & 15 & 8,36 & 1,70 & \multirow{2}{*}{0,99} \\
\hline & ESF & 71 & 5,79 & 3,51 & & 19 & 8,35 & 1,53 & \\
\hline \multirow{2}{*}{ Orientação familiar } & UBT & 303 & 4,97 & 2,65 & \multirow{2}{*}{0,12} & 15 & 7,57 & 2,10 & \multirow{2}{*}{0,15} \\
\hline & ESF & 71 & 5,51 & 2,65 & & 19 & 8,55 & 1,64 & \\
\hline \multirow[t]{2}{*}{ Orientação comunitária } & UBT & 187 & 3,04 & 2,31 & \multirow[t]{2}{*}{$0,00^{*}$} & 15 & 6,67 & 2,39 & \multirow[t]{2}{*}{0,15} \\
\hline & ESF & 62 & 6,93 & 2,59 & & 19 & 5,61 & 1,57 & \\
\hline Essencial & $\begin{array}{l}\text { UBT } \\
\text { ESF }\end{array}$ & - & $\begin{array}{l}6,32 \\
6,29\end{array}$ & $\begin{array}{l}0,48 \\
0,34\end{array}$ & 0,95 & - & $\begin{array}{l}6,98 \\
6,46\end{array}$ & $\begin{array}{l}0,64 \\
0,65\end{array}$ & 0,63 \\
\hline Derivado & $\begin{array}{l}\text { UBT } \\
\text { ESF }\end{array}$ & - & $\begin{array}{l}3,99 \\
6,22\end{array}$ & $\begin{array}{l}0,42 \\
0,28\end{array}$ & 0,21 & - & $\begin{array}{c}7,11 \\
7,08\end{array}$ & $\begin{array}{l}0,21 \\
0,64\end{array}$ & 1,00 \\
\hline Geral & $\begin{array}{l}\text { UBT } \\
\text { ESF }\end{array}$ & - & $\begin{array}{l}5,85 \\
6,28\end{array}$ & $\begin{array}{l}0,43 \\
0,43\end{array}$ & 0,51 & - & $\begin{array}{l}7,01 \\
6,62\end{array}$ & $\begin{array}{l}0,54 \\
0,61\end{array}$ & 0,64 \\
\hline
\end{tabular}

Fonte: Elaboração própria

${ }^{\star}$ Teste ' $t$ ', de Student, para diferença de média; diferença estatisticamente significativa $(p<0,05)$

Negrito: Média de escore acima de 6,6 (ponto de corte para valor considerado alto)

Para os usuários dos distintos serviços, o valor do Escore Geral da APS, que mede o grau de orientação para a atenção primária, foi considerado baixo nos dois modelos de serviço. A ESF apresentou um escore maior, mas sem diferença significativa em relação às unidades tradicionais. Com relação aos atributos, isoladamente, chama a atenção a predominância dos escores abaixo do valor 6,6 em ambos os serviços. Em geral, os dados apresentam melhor desempenho para as crianças que têm a ESF como fonte regular de cuidado, no entanto, apenas nos atributos Longitudinalidade, Coordenação-Sistema de Informação e Orientação comunitária houve diferença significativa quanto às UBT no que tange aos dados coletados pelo PCATool. As UBT apresentam maiores disponibilidades de serviços (diferença significativa) e melhores serviços prestados do que as UBSF. Destaca-se o baixo desempenho dos cuidados direcionados à família pelas equipes da ESF, na opinião das mães, pelo seu papel estruturante nesse modelo de APS. 
Diferentemente da opinião dos usuários do Distrito Federal, estudos utilizando o PCATool, comparando distintos modelos de serviços - e realizados em outros municípios brasileiros, entre eles, Porto Alegre (OLIVEIRA, 2007), São Paulo (ellas et AL., 2006), Montes Claros (LEÃO; CALDEIRA; OLIVEIRA, 2011) e nove outros municípios da região Centro-Oeste (VAN STRALEN ET AL., 2008) -, apontaram melhores resultados dos atributos da APS nos serviços prestados pelas unidades do saúde da família, em comparação com os tradicionais, na avaliação dos usuários.

A insatisfação dos usuários com os serviços da atenção primária também foi demonstrada em um estudo avaliativo realizado por meio do PCATool, em 62 municípios do estado de São Paulo, comparando as unidades do PSF com os modelos tradicionais. $\mathrm{O}$ mesmo estudo demonstra que, na opinião dos profissionais, a ESF apresentou um melhor desempenho (IBANEZ ET AL., 2006).

Já se sabe que a presença desses atributos nos serviços de atenção primária é fundamental para garantir seus resultados e a qualidade dos serviços à população. Para Facchini et al. (2006), a ausência de um ou mais desses atributos pode ser a causa responsável pela heterogeneidade da qualidade da ESF.

No geral, observa-se que os profissionais costumam avaliar os seus serviços melhor do que os usuários, e, no Distrito federal, isso não foi diferente (ELIAS ET AL., 2006). Na opinião daqueles, os seus serviços vêm desempenhando esses atributos de forma satisfatória, como mostra a predominância dos escores acima do valor 6,6. O grau de orientação para a APS também foi avaliado como adequado para os dois modelos de APS.

$\mathrm{Na}$ avaliação dos atributos, isoladamente, apenas as dimensões Acessibilidade e Integralidade - serviços disponíveis apresentaram escores inferiores ao ponto de corte. Entretanto, para a ESF, também se observou que o atributo Orientação comunitária obteve baixo escore na avaliação dos seus profissionais. Destaca-se, também, o baixo desempenho do atributo Orientação comunitária, considerado estruturante da ESF, na avaliação dos seus profissionais.

O gráfico 1 apresenta a síntese dos resultados na opinião dos cuidadores de crianças e dos profissionais que trabalham nos dois modelos de serviços da APS no Distrito Federal.

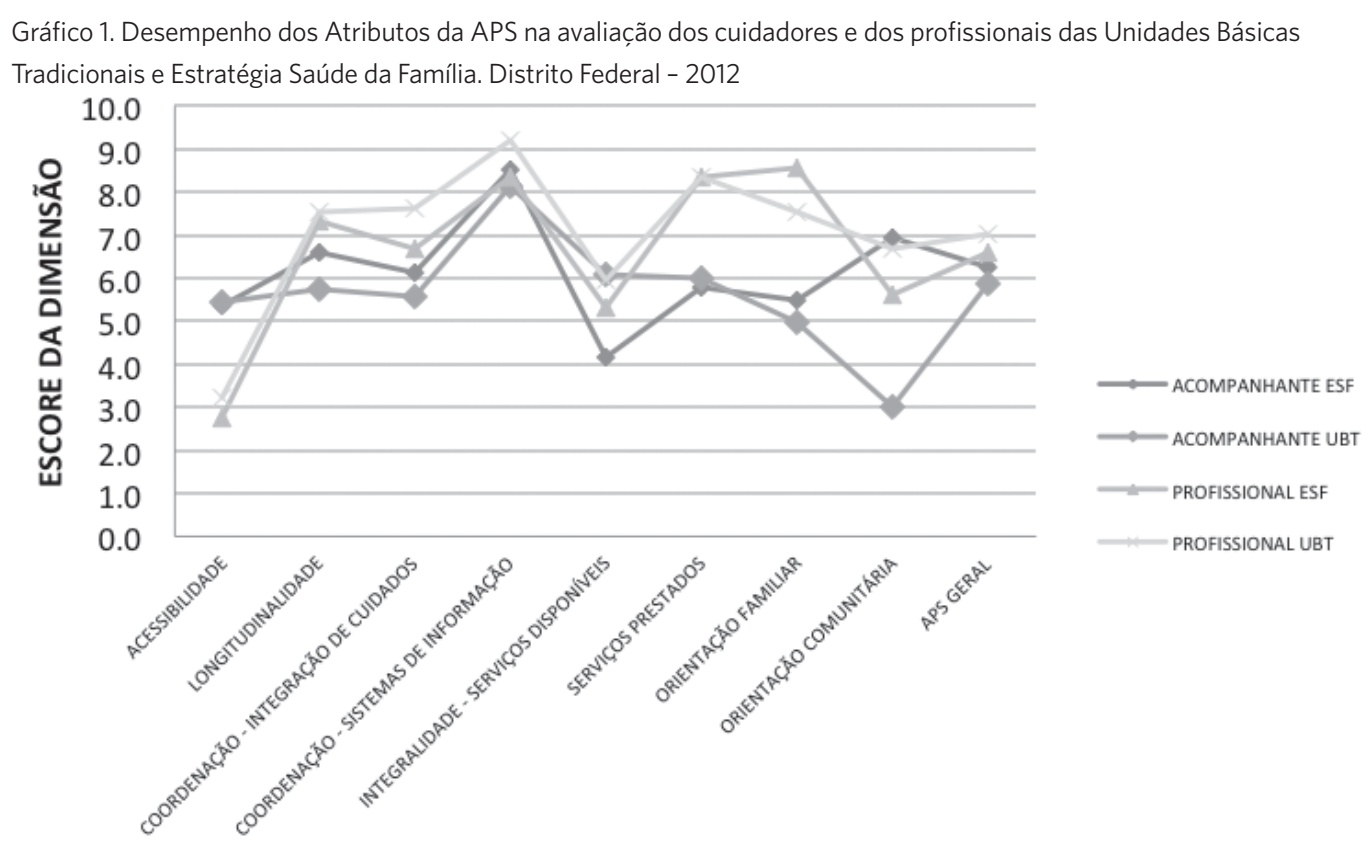


Com o intuito de ampliar a discussão final, faremos um diálogo sobre os resultados, mediante a combinação das informações colhidas nos dois métodos. No grau de afiliação, a ESF apresentou melhor desempenho. Assim, na percepção das mães, os serviços da ESF foram identificados, com mais facilidade, como fonte regular de atenção às suas crianças. Muito embora a identificação de um serviço como referência para o usuário esteja contemplada no atributo longitudinalidade, optamos por fazer uma avaliação dos resultados do Grau de afiliação.

Esses resultados se aproximam do que é preconizado nas suas bases organizativas, quanto à: vinculação territorial; organização da oferta de serviço pelas ações programáticas; proximidade da equipe com as famílias; e convivência dos profissionais, nos cotidianos das suas práticas, com os seus membros e em seu entorno (comunidade). E os resultados nas UBT nos fazem compreender que essas unidades se configuram de forma diferente, por não possuírem uma adstrição de clientela, pela extensão territorial, pela dispersão da população e, ainda, pelo formato do seu processo organizativo na oferta de ações e serviços de saúde, nos quais predomina a livre demanda de atendimento.

Em seus estudos, Elias et al. (2006), Oliveira (2007) e Ibanez et al. (2006), ainda que não mencionem diretamente a questão da afiliação dos usuários aos seus serviços, remetem ao vínculo entre eles e os profissionais de saúde em seus territórios trabalhados. Isso colabora com nossos achados, na medida em que a questão do vínculo é expressa por várias falas dos sujeitos entrevistados neste estudo.

Os estratos de falas abaixo revelam a identificação da unidade como um espaço do cuidado e nos instigam a pensar sobre a afiliação.

[...] a gente até teve uma mãe que morava na área. Fizemos o pré-natal dela, o Crescimento e Desenvolvimento (CD) do filho até um ano. Ela mudou da área, e o agente de saúde disse: 'Fulana, agora você é da equipe oito'. E a mãe respondeu: 'Não, eu quero continuar aqui, eu quero a Dra. P, eu quero a Enfermeira M!'. Ai, a agente comunitária disse: ' ela não quer sair do meu pé, ela me procura, ela me cobra as consultas do fiIhinho dela. (E1 ESF).

[...] infelizmente, tem aquelas mães, que não são dos nossos endereços, que vêm uma vez ou outra, vêm só quando necessita. Mesmo assim, elas vêm com aquela certeza de que ela vai encontrar apoio, entende? (E3 UBT).

Em síntese, pode-se afirmar que a identificação dessas unidades por parte das mães se configura em uma relação positiva do ato de cuidar. $\mathrm{O}$ acesso como porta de entrada parece adequado, no entanto, o acesso às ações e aos serviços ainda está complicado, na percepção dos usuários e também dos profissionais dos distintos serviços. Esse resultado é semelhante ao encontrado por Macinko et al. (2006), em Petrópolis, por Chomatas (2008), em Curitiba, e por Elias et al. (2006), no município de São Paulo. Em São Paulo, Elias et al. (2006) sugerem dificuldades estruturais na oferta de serviços, para além das de organização interna das unidades, observadas nos rígidos e limitados horários de funcionamento das UBS, principalmente nas grandes metrópoles, prejudicando o acesso de uma parcela da população trabalhadora aos serviços de atenção primária. Na opinião dos profissionais entrevistados no DF, a limitação do horário de funcionamento das unidades também foi considerada um dos fatores que dificultam o acesso, assim como a organização da agenda de atendimento das equipes da ESF, que disponibilizam um horário semanal para $\mathrm{o}$ atendimento à população infantil, limitando o acesso, principalmente, para assistência à criança doente.

No que diz respeito ao entendimento dos profissionais acerca do acesso, os entrevistados apontam para o direito dos pacientes de circular nas Unidades de Estratégia de Saúde da Família (Uesf). O acesso também se reflete nas formas de organização das UBS, no tocante ao acolhimento e à capacidade da resposta do serviço às necessidades do usuário. 
De qualquer forma, as falas que se seguem nos faz refletir que a compreensão do acesso, no entendimento dos profissionais, restringe-se à chegada aos serviços, atrelada às dinâmicas organizacionais dos mesmos.

[...] O direito de ir e vir, de ser atendido naquele momento que, provavelmente, a paciente acha que deveria. Então, acesso, acessibilidade, é o contato que ele teria com a unidade, (o direito) de entrar e sair. (E2 ESF).

[...] aqui no centro de saúde? Eu entendo o acesso do usuário, assim, como um lugar que ele procura, que tem que ter a facilidade de atendimento, uma facilidade no acolhimento, para a gente tentar fazer o possível para ajudá-lo no que ele precisa. Para mim, é isso, o acesso dele aqui é isso, livre, para que a gente possa ajudá-lo em alguma coisa, na necessidade dele. (E3 UBT).

[...] acesso é quando o paciente vem ao serviço e ele é acolhido. Não necessariamente ele tem que ter a consulta imediata, no acolhimento que é feito, se há uma necessidade de atendimento imediato, se você pode agendar aquele paciente, colocando ele, mesmo na Atenção Básica, dentro ali de um protocolo de classificação. (M1 ESF).

Essa dinâmica funcional é impeditiva e impõe barreiras a um diálogo a ser estabelecido entre as demandas e expectativas da população, por um lado, e a sensibilidade dos serviços de estarem abertos a esse diálogo, por outro. Entre os fatores facilitadores para assegurar o acesso, os entrevistados revelam que a presença do Agente Comunitário de Saúde (ACS) na ESF faz a diferença para a sua extensão, uma vez que estabelece a ponte entre os serviços e as famílias às quais estão vinculadas as suas áreas de atuação.

[...] Não existe dificuldade de acesso. A gente tem a captação pelos agentes comunitários, usando a ficha das crianças. Temos a monitorização da data do parto, justamente para a gente poder captar e avaliar o RN [recém-nascido] e a puérpera o mais precoce. (M1 ESF).

[...] A criança, nesse centro de saúde, tem acesso [...] a gente faz, assim, o cronograma de acordo com o acompanhamento do $C D,[. .$.$] então, esse$ acesso à saúde, eu falo que é privilegiado, a gente não deixa a criança desamparada aqui. (E2 UBT).

Os extratos das falas nos remetem a duas concepções do acesso e da acessibilidade: $o$ primeiro, como porta de entrada aos serviços de saúde; e o segundo, como características organizativas dos seus serviços. No que afirma Starfield (2002), acessibilidade e acesso podem ser medidos a partir dos argumentos expressos tanto pela população quanto pelas unidades, que têm a responsabilidade pela oferta e pela prestação dos serviços.

Esses relatos auxiliam na compreensão de que, diferentemente do que apresenta a pesquisa, no tocante ao acesso, é preciso pensar naquilo que aponta Sousa (2007, P. 55):

o acesso como a inter-relação estabelecida entre os indivíduos e a família e a comunidade, gestores e equipes do PSF, permeada pelo vínculo e pela corresponsabilidade, no exercício permanente de geração de oportunidades e capacidades entre os sujeitos no processo do cuidar da saúde - doença - morte - como expressões de respeito, autonomia e participação.

Verifica-se que os resultados desse estudo, para se aproximarem dos pressupostos defendidos pelos autores e mencionados nas falas dos entrevistados, provocam pensar que $o$ acesso no DF ainda se constitui uma lacuna.

Para o cuidado ao longo do tempo, a ESF foi considerada a melhor opção na avaliação dos cuidadores. Já para os profissionais, os dois serviços apresentaram valores satisfatórios. Para esse atributo, é importante considerar a percepção dos cuidadores e dos profissionais, já que a essência da longitudinalidade é uma relação que se estabelece ao longo do tempo entre os indivíduos e um profissional ou a equipe de saúde. 
A presença da equipe da ESF junto à população e a responsabilidade do cuidado estendido aos indivíduos e à família em seus ciclos de vida facilitaram a formação do vínculo com essas mães.

[...] a gente vê crescer, vê a gestação, vê a criança nascer, vê o desenvolver dela, vê as dificuldades da mãe com a pega, então, a gente acaba acompanhando sempre, porque está na nossa área, e, como a gente tem muito tempo na mesma área... A equipe não muda muito, é a mesma equipe há três anos, então, fica mais fácil. (E3 ESF).

Para os profissionais das UBT, o tempo do profissional no serviço favorece a extensão do vínculo.

[...] as mães conhecem a gente. Elas já chegam aqui e querem falar com a enfermeira, porque as mães já me conhecem, eu trabalho aqui há 20 anos, [...] eu cobro esse retorno das mães, eu quero saber o que aconteceu. Então, a criança, até os que fazem acompanhamento especializado no hospital de câncer, elas retornam, elas falam como é que estão. (E3 UBT).

O acompanhamento do Crescimento e Desenvolvimento (CD) da criança é visto como uma estratégia para o estabelecimento do vínculo, ao mesmo tempo que se exercita a responsabilidade de toda a equipe.

[...] Primeiro, que eles vão ter essas consultas, pelo menos nos primeiros anos de vida, garantido, só pra acompanhar mesmo, e, quando tiver alguma coisa, tem que procurar [...] o agente de saúde faz o contato, o auxiliar de enfermagem participa, pelo menos, da triagem e da avaliação inicial da criança, a enfermeira e o médico fazem as consultas alternadas do crescimento e desenvolvimento. (M2 ESF).

Por outro lado, a pouca permanência da família na sua área está associada à dificuldade de adesão ao cuidado longitudinal e de criação do vínculo com a equipe cuidadora.
[...] Seria ótimo trabalhar com uma população que não mudasse nunca de endereço, porque a gente conseguiria ter maior domínio, maior conhecimento, maior vínculo, mas a realidade que a gente tem hoje é uma população muito mutável. (E4 ESF).

[...] bem, só no acompanhamento do CD que a gente vê que a falha maior é daqueles que moram mais longe e não têm condições de fazer o acompanhamento, [...] as que não fazem direito o CD são realmente as de fora. (E1 UBT).

De acordo com o Ministério da Saúde, o acompanhamento da criança será feito visando a estreitar o vínculo da criança e da família com os serviços de saúde, propiciando oportunidade de abordagem de promoção da saúde, prevenção de problemas e cuidado em tempo oportuno. Assim, constitui-se como uma estratégia importante para uma relação terapêutica mútua e duradoura entre os cuidadores e os profissionais. Nessa direção, observou-se que os profissionais das UBS do Distrito Federal percebem o acompanhamento do $\mathrm{CD}$ como estratégia de cuidado ao longo do tempo.

O cuidado longitudinal se constitui em um atributo essencial no contexto do cuidado infantil, no sentido de que a relação com uma fonte regular de cuidados implica a sua escolha para a atenção em cada novo problema. No entanto, e de acordo com Starfield (2002), os atributos se relacionam entre si, sendo necessário que essa fonte habitual de atenção seja integral e coordenadora. Apesar de sua importância como atributo estruturante da APS, a longitudinalidade não apresenta respostas para todas as demandas. A articulação com outros níveis de atenção e a intersetorialidade precisam estar presentes (CUNHA; ELENICE; GIOVANELLA, 2013). Nesse caso, a maior extensão da longitudinalidade na ESF também vai ao encontro dos seus princípios organizativos, como discutido no item afiliação.

A avaliação do leque de serviços disponíveis, pelos usuários, também não foi satisfatória. Porém, nessa dimensão, o desempenho das UBT foi sensivelmente superior ao das equipes da ESF. Nesse caso, também os 
profissionais avaliaram que a oferta de serviços não está adequada. A prática da integralidade implica as respostas, por parte dos serviços, das necessidades de saúde dos indivíduos. No geral, as mães avaliaram que nem sempre esses serviços atendem às necessidades mais comuns das suas crianças.

O melhor desempenho das UBT, provavelmente, está associado às melhores estruturas, à presença diária de pediatras nas unidades e à melhor oferta de ações e atividades direcionadas às crianças, no comparativo com a ESF. Na ESF, algumas unidades não disponibilizam vacinas, e a inadequação das estruturas físicas impossibilita atividades de promoção em grupo. A utilização dos espaços comunitários nem sempre é adequada para atividades coletivas, como foi observado pelos pesquisadores. A organização da agenda, disponibilizando um único período para atendimento, também pode ser um fator limitador para a assistência à criança quando adoece.

As falas desses profissionais apresentam aspectos dificultadores para a extensão desse atributo nos dois modelos de serviços, o que nos remete a questões estruturais e organizacionais, como também a dificuldades para além das de organização interna.

[...] acho um absurdo a falta de vacina. Pelo fato de ser zona rural e o acesso ser complicado, 0 paciente tem que se deslocar mil e poucos quilômetros até uma pista para poder vacinar. É um trabalho integral pela metade, porque, realmente, vacina é primordial na saúde da criança. (E2 ESF).

[...] não conseguimos fazer as palestras educativas porque não tem local nem espaço [...] a unidade tem quatro equipes. $O$ espaço é muito pequeno, até pra todo mundo atender, não tem espaço pra ninguém. (E3 ESF).

[...] ontem, pedi um raio-X de seio da face. Falei para a mãe: ' tenta fazer na Policlínica, que é mais rápido, e já traz para a gente ver. Vou deixar até seu prontuário aqui'. E à tarde ela veio: 'sim, cadê o exame'? Ela fez, mas só dia vinte e tantos vai ficar pronto. É complicadíssimo! (M4 UBT).

[...] no nível da Atenção Básica, como médica de família, a gente tem tentado fazer o que é possível, o que é viável. Mas, já para o nível secundário e terciário, é preciso ter uma parceria maior, uma regulação melhor. (M1 ESF).

Diante desses relatos, conclui-se que o desafio para o alcance da integralidade está, principalmente, relacionado a problemas estruturais e organizacionais nos serviços avaliados. Por outro lado, a identificação das necessidades do indivíduo está relacionada à compreensão do conceito amplo de saúde, e a ESF tem maior reconhecimento dos problemas biológicos, psicológicos e sociais que causam a doença, com melhor percepção da compreensão de integralidade. Na fala desses profissionais, a integralidade se expressa na forma como eles vivenciam as suas práticas:

[...] A integralidade, para mim, está muito dentro do atendimento humanizado, de você ver desde a questão de saúde física da criança até os fatores envolvidos, que é a relação familiar, o ambiente que ela vive, quem é que cuida, questão da atenção a outras variáveis do atendimento. [...] as visitas domiciliares, no intuito de ver quando a gente detecta algum caso possível de violência física ou psicológica. [...] Para mim, a integralidade é isso, não só a questão do $C D$, da criança vir e acompanhar a saúde física, mas as outras variáveis da saúde, saúde mental e o ambiente, a estrutura da família também. (E4 RFII).

[...] Integralidade? A gente tenta, na verdade, a consulta muitas vezes. Com quinze minutos, é difícil você falar muitas coisas. Integralidade é ver alguma coisa de doença, se a criança tem alguma doença e falar sobre prevenção. O ideal, na verdade, era falar sobre prevenção. Então, se o menino está um pouco mais gordinho, e tudo, então você fala e dá um pouco mais de atenção. Aí, você vai falar sobre o problema da obesidade, do sedentarismo, hipertensão arterial. Você fala dos cuidados que a mãe tem que ter, cuidados devidos às intoxicações e tudo. (M4 UBT). 
Para Sousa, Erdmann e Mochel (2010), a integralidade, como princípio da política de saúde, remete à compreensão de que os fatores que interferem na saúde da criança são amplos e perpassam outros setores além da saúde. O modelo médico hegemônico, ainda prevalente nas práticas em saúde, expressa-se por ações prioritariamente curativas, centradas na doença e sem a complementaridade das ações de proteção, promoção e prevenção de agravos à saúde infantil. Observou-se que o enfoque forte na prevenção e na atenção às doenças dos profissionais das UBT reflete o modelo médico hegemônico dominante, repercutindo nas suas práticas de saúde. Por outro lado, para os profissionais da ESF, já se observa um novo olhar, em todas as dimensões do cuidado e na percepção das necessidades da criança, da família e da comunidade.

Como fator facilitador, os serviços da ESF têm a presença do ACS e a visita domiciliar:

[...] talvez, em um sistema tradicional, você vai fazer um cuidado só dentro do consultório e orientar só o que você está vendo. O cuidado integral envolve o agente comunitário que está lá todo mês, e ele sabe te dizer 'essa criança é bem cuidada, essa criança não é. Essa criança a mãe não faz a vacina'. Você visualiza a criança além do que a mãe fala. Quando você vai à casa você consegue enxergar coisas que elas te dizem que é o ideal, mas não é. (E1 ESF).

A insatisfação dos usuários com a oferta de serviços nos dois modelos reflete o não cumprimento do atributo na sua integralidade, no baixo escore atribuído (de comum acordo com os profissionais) e nas suas falas, quando remetem à situação de suas práticas, das questões estruturais e da rede de serviços da Secretaria de Saúde. Principalmente no caso da ESF, que já incorpora a compreensão do conceito de integralidade do cuidado, tendo na estrutura dos seus serviços fator impeditivo para a prática efetiva desse atributo.

Em coordenação dos cuidados, os escores obtidos foram baixos em ambos os serviços, segundo os usuários. Para a coordenação cumprir com os seus princípios, precisa garantir a continuidade da atenção nos demais pontos de serviços, tendo, na sua essência, a informação e a utilização da informação e de uma rede de serviço capaz de dar respostas às demandas de saúde da população. Na percepção dos profissionais, existem vários desafios para a coordenação, em diferentes contextos.

[...] Existe uma dificuldade grande, porque, primeiro, a referência é complicada. É difícil porque falta vaga, falta profissional. Quando o problema precisa ser enviado para o nível secundário, às vezes, se perde, o cuidado fica interrompido, porque a pessoa, às vezes, não tem acesso nunca, fica um ano ou anos esperando. (M2 ESF).

[...] a gente não tem aquele feedback, a gente encaminha a criança e... Muitas vezes, eu pergunto pra mãe: 'e aí, teve a consulta com o neurologista? A senhora já pediu?'. 'Não ou já teve', mas não foi anotado nada. 'Não, ele passou exame, fez isso...', quando a mãe sabe falar, porque, quando a mãe não sabe, você fica sem saber de nada. (M4 UBT).

Sem adequada coordenação do cuidado, a longitudinalidade perde muito do seu potencial, a integralidade fica comprometida, e o primeiro contato torna-se um ato meramente administrativo. Os problemas relacionados à coordenação dos cuidados em saúde, à fragmentação da rede assistencial e à falta de comunicação entre os níveis de serviços se constituem em desafios para os sistemas de saúde no DF, na perspectiva de uma APS coordenadora do seu sistema de saúde.

\section{Conclusão}

Nosso estudo partiu da análise da presença e da extensão dos atributos da APS, sendo direcionado ao processo de cuidado integral à saúde da criança, comparando-o, nas UBST e na ESF, no âmbito do Sistema Único de Saúde do DF.

De início, é clara a diferença existente entre a avaliação dos usuários e dos profissionais de 
saúde. Tanto na avaliação geral da APS como em quase todas as suas dimensões, os usuários a consideram menos positiva nos dois modelos de serviços.

Percebe-se que o exercício das práticas dos profissionais, tanto das unidades tradicionais quanto das equipes da ESF, e a compreensão sobre a base conceitual e operacional do acesso, da longitudinalidade, da integralidade e da coordenação apresentam-se difusos, confusos e, às vezes, numa relação de dependência com as formas de organização balizadas por conceitos tradicionais.

Assim, no cotidiano do trabalho, observa-se que os profissionais dos dois modelos vêm se esforçando no sentido de facilitar o acesso como porta de entrada e o cuidado ao longo do tempo, e que considera-se potencialidades. Entretanto, nas práticas dos profissionais, verificam-se fragilidades no acesso aos cuidados disponíveis, na coordenação do cuidado e na integralidade.

Também observa-se que, no conjunto de ações de intervenção direcionadas à criança, as equipes da ESF já a visualizam no seu contexto familiar e a família como o sujeito da atenção. Nesse sentido, destaca-se a extensão do cuidado no domicílio, que tem se constituído como espaço relevante para o desenvolvimento de ações de cuidado e promoção de saúde, de reconhecimento dos problemas não apenas no âmbito biológico, mas, também, psicológico e social, ampliando o seu leque de serviços e, ao mesmo tempo, favorecendo o fortalecimento do vínculo entre a família e seus cuidadores.

\section{Referências}

BRASIL. Ministério da Saúde. Secretaria de Atenção à Saúde. Departamento de Atenção Básica. Manual do Instrumento de Avaliação da Atenção Primária à Saúde: primary care assessment tool PCATool-Brasil. Brasília: Ministério da Saúde, 2010.

Ministério da Saúde. Secretaria de Atenção à Saúde. Departamento de Atenção Básica. Política Nacional de Atenção Básica. Brasília, DF: Ministério da Saúde, 2012
No tocante às facilidades, há algumas causas que circulam entre as relações estabelecidas na oferta e na demanda das ações e dos serviços de saúde, onde os ACS apresentam-se como mediadores dessa relação. Estes, passando a atuar como facilitadores do acesso, em particular. O fato de os ACS circularem com mais 'propriedade' nos territórios de atuação das ESF é fator que facilita a coordenação e o desenvolvimento na busca da integralidade.

No geral, a força de orientação para a APS no DF foi considerada baixa, na opinião dos usuários dos distintos serviços, porém, mais favorável à ESF, sem diferença estatisticamente significativa em relação às UBST. Entretanto, apesar de a amostra do estudo ser representativa de duas RA, com modelos distintos de APS, não se pode estender os resultados para as demais RA, muito embora seja possível que as demais RA possam repetir as conclusões apresentadas. Para finalizar, considera-se importantes os estudos científicos que possam contribuir para uma melhor avaliação da APS em outras RA do DF, contribuindo, assim, para subsidiar a construção de um modelo consistente de APS.

\section{Agradecimentos}

Luciani Martins Ricardi, que participou como pesquisadora de campo e contribuiu para a discussão dos resultados. Rackynelly Alves, pela sua valiosa análise dos dados quantitativos.
CHOMATAS, E. R. V. Avaliação da Presença e extensão dos Atributos da Atenção Primária à Saúde no Município de Curitiba em 2008. 2009. 95 f. Dissertação (Mestrado em Epidemiologia) - Universidade Federal do Rio Grande do Sul, Porto Alegre, 2009.

CUNHA, E. M.; GIOVANELLA, L. Longitudinalidade/ continuidade do cuidado: identificando dimensões e variáveis para a avaliação da Atenção Primária no contexto do sistema público de saúde brasileiro 
[internet]. Ciênc. Saúde Colet., Rio de Janeiro, v. 16, supl. 1, 2011. Disponível em: <http://www.scielo.br/scielo. php?pid=S1413-81232011000700036\&script=sci_arttext>. Acesso em: 18 dez. 2014.

ELIAS, P. E. et al. A Atenção Básica em Saúde: comparação entre PSF e UBS por estrato de exclusão social no município de São Paulo. Ciênc. Saúde Colet., Rio de Janeiro, v. 11, n. 3, p. 633-641, 2006

FACCHINI, L. A. et al. Desempenho do PSF no Sul e no Nordeste do Brasil: avaliação institucional e epidemiológica da Atenção Básica à Saúde. Ciênc. Saúde Colet., Rio de Janeiro, v. 11, n. 3, p. 669-681, 2006.

HARZHEIM, E. et al. Consistência interna e confiabilidade da versão em português do instrumento de avaliação da atenção primária (PCATool-Brasil) para serviços de saúde infantil. Cad. Saúde Pública, Rio de janeiro, v. 22, n. 8, p. 1649-1659, 2006.

IBANEZ, N. et al. Avaliação do desempenho da atenção básica no Estado de São Paulo. Ciên. Saúde Colet., Rio de Janeiro, v. 11, n. 3, p. 683-703, 2006.

LEÃO, C. D. A. ; CALDEIRA, A. P.; OLIVEIRA, M. M. C. Atributos da atenção primária na assistência à saúde da criança: avaliação dos cuidadores. Rev. Bras. Saúde Mater. Infant., Recife, v. 11, n. 3, p. 323-334, 2011.

MACINKO, J. et al. Evaluation of the impact of the family health program on infant mortality in Brazil, 1990-2002. Journal of Epidemiology and Community Health, Londres v. 60, n. 1, p. 13-19, 2006.

MENDES, E. V. O cuidado das condições crônicas na Atenção Primária à Saúde: o imperativo da consolidação da Estratégia da Saúde da Família. Brasília, DF: Organização Pan-Americana da Saúde, 2012.

MINAYO, M. C; SANCHEZ, O. Quantitative and Qualitative Methods: Opposition or Complementarity? Cad. Saúde Pública, Rio de Janeiro, v. 9, n. 3, p. 239-262, 1993.

OLIVEIRA, M. M. C. Presença e extensão dos atributos da Atenção Primária à Saúde entre os serviços de Atenção
Primária em Porto Alegre: uma análise agregada. 2009. $118 f$. Dissertação (Mestrado em Epidemiologia) - Universidade Federal do Rio Grande do Sul Porto Alegre, 2007.

\section{ROMERO, R. V; COLLANTES, J. M; RAMIREZ, N. A.} Evaluación rápida del desempeño de la red pública de servicios de salud de Suba en el logro de los atributos de la Atención Primaria de Salud-APS. Rev. gerenc. políticas salud, Bogotá, v. 8, n.16, p. 165-190, 2009.

STARFIELD, B. Atenção Primária: equilíbrio entre necessidades de saúde, serviços e tecnologia. Brasília: UNESCO, Ministério da Saúde, 2002.

STARFIELD, B.; XU, J.; SHI, L. Validating the Adult Primary Care Assessment Tool. The Journal of Family Practice, Parsippany, v. 50, n. 2, p. 161-175, 2001.

SOUSA, M. F; HAMANN, E. M. Programa Saúde da Família no Brasil: uma agenda incompleta? Ciên. Saúde Colet. Rio de Janeiro, v. 14, supl. 1, p. 1325-1335, 2009.

SOUSA, M. F. Programa Saúde da Família no Brasil: análise da desigualdade no acesso à Atenção Básica. Brasília, DF: Universidade de Brasília, Departamento de Ciência da Informação e Documentação, 2007.

\section{SOUSA, F. G. M; ERDMANN, A. L; MOCHEL, E. G.}

Modelando a integralidade do cuidado à criança na Atenção Básica de Saúde [internet]. Rev. Gaúcha Enferm. v. 31, n. 4, p. 701-707, 2010. Disponível em: <http:// www.scielo.br/scielo.php?script=sci_arttext\&pid $=$ S1983-14472010000400013 $>$. Acesso em: 20 dez. 2014.

VAN STRALEN, C. et al. Percepção dos usuários e profissionais de saúde sobre atenção básica: comparação entre unidades com e sem saúde da família na região do CentroOeste do Brasil. Cad. Saúde Pública, Rio de janeiro, v. 24, supl. 1, p. 148-158, 2008.

Recebido para publicação em março de 2014

Versão final em agosto de 2014

Conflito de interesse: inexistente

Suporte financeiro: não houve 